Int. J. Dev. Biol. 61: 159-164 (2017)

doi: $10.1387 / \mathrm{ijdb} .160388 \mathrm{LB}$

\title{
The SUMO system in Caenorhabditis elegans development
}

\author{
LIMOR BRODAY* \\ Department of Cell and Developmental Biology, Sackler School of Medicine, Tel Aviv University, Tel Aviv, Israel
}

\begin{abstract}
SUMO, a small ubiquitin-like modifier, is a highly conserved post translational modification and a central regulatory system in eukaryotes. Sumoylation modulates the activities of multiple proteins, mainly in the nucleus, such as transcription factors, chromatin modifiers, and proteins involved in DNA replication and repair. However, SUMO also modifies substrates in the cytoplasm, mitochondria, plasma and ER membrane. This review summarizes our current knowledge on the functions of sumoylation in C. elegans development. SUMO modification is highly reversible and several examples described here establish its function as a molecular switch during embryogenesis and postembryonic organogenesis.
\end{abstract}

KEY WORDS: SUMO, post translational modifications, C. elegans

\section{Introduction}

Sumoylation is a reversible post-translational modification that modulates the localization, stability, activity and the interactions of the target protein with other proteins and cellular components (Flotho and Melchior, 2013). Sumoylation is involved in the regulation of numerous cellular functions that participate in developmental processes such as cell division, cell fate decisions, differentiation and morphogenesis. Proteins in the nucleus, cytosol and the plasma membrane can be targets of SUMO. Higher eukaryotic genomes encode for several SUMO paralogs with different functions, while a single gene encodes for SUMO in yeast and invertebrate model organisms. Although this machinery resemblesubiquitination in many aspects, the effect of sumoylation is different from ubiquitination and usually, as described in this review, is substrate specific. One of the main characteristics of the SUMO machinery is its transient nature making it difficult for classical biochemical analysis but an excellent system to decode using model organisms. I will focus on the current main knowledge about the function of SUMO in $C$. elegans development.

\section{The components of the SUMO system in C. elegans}

SUMO. C. elegans encodes only a single SUMO protein called SMO-1 that closely resembles mammalian SUMO1. SMO-1 is essential for viability in $C$. elegans and knockdown caused sterility (due to a maternal product) and lethality (Broday et al., 2004; Jones et al., 2002). Overexpression of SUMO also impaired normal development, indicating that regulation of SUMO levels and probably the activity of the entire sumoylation system is critical for normal development (Rytinki et al., 2011). The distinct enzymatic pathway that conjugates SUMO to target proteins is highly conserved (Flotho and Melchior, 2013).

E1. A heterodimeric E1 protein consisting of the UBA-2 and AOS-1 proteins and activates SUMO in a two-step reaction that involves ATP hydrolysis and forms a thioester bond with the GlyGly motif in the C-terminus of SUMO. The thioester-charged E1 enzyme interacts with the E2(UBC9) and SUMO is subsequently transferred to the E2 enzyme.

E2. The single E2 SUMO-conjugating enzyme, ubiquitin-like conjugating enzyme 9 (UBC-9), has a key function in the system. In addition to carrying the activated SUMO to the substrate, it recognizes SUMO acceptor site/s on the substrate and directly conjugates SUMO onto a target lysine. UBC-9 is essential for $C$. elegans viability (Jones et al., 2002).

SUMO E3 ligase, GEI-17. Unlike the E3 ligases of the ubiquitin system, SUMO E3 ligases are not essential components of the sumoylation reaction in vitro and few proteins are known to be efficiently sumoylated in the absence of E3 ligases also in vivo (Flotho and Melchior, 2013). Proteins that are considered as SUMO E3 ligases have been shown to enhance specificity and accelerate the sumoylation process in vivo (mainly Siz/PIAS and RanBP2 (Gareau and Lima, 2010). GEI-17, a PIAS domain protein, has been shown in $C$. elegans to have multiple functions mainly during embryogenesis. It acts as an E3 ligase for MUS101 sumoylation (Holway et al., 2005), regulator of TBX-2 (Milton

Abbreviations used in this paper: $\mathrm{DCC}$, dosage compensation complex; ER, endoplasmic reticulum; SUMO, small ubiquitin-like modifier.

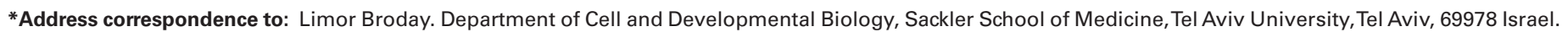
Tel: 972-3-6406653. Fax: 972-3-6407432. e-mail: broday@post.tau.ac.il
}

Accepted: 23 November, 2016.

ISSN: Online 1696-3547, Print 0214-6282 
and Okkema, 2015; Roy Chowdhuri et al., 2006) and mediates sumoylation of POLH-1 to protect it from CRL4-Cdt2-mediated degradation in early embryos during DNA-damage response (Kim and Michael, 2008; Roerink et al., 2012). In addition, gei-17 (as well as the SUMO protease ulp-1) was identified in genomewide RNAi screen for genes sensitizing embryos to MMS (methyl methane sulphonate) and gei-17(RNAi) led to abundant RAD51 foci in embryos treated with MMS, indicative of replication stress (Roerink et al., 2012). GEl-17 was also shown to be required for telomere positioning in early embryos and gei-17(RNAi) resulted in delocalization of telomeres from the nuclear periphery (Ferreira et al., 2013). GEl-17 mediates accumulation of SUMO conjugates on the metaphase plate during the first embryonic cell division and enhance the sumoylation of AIR-2 (Aurora B orthologue) in vitro (Pelisch et al., 2014).

SUMO proteases. Sumoylation is a highly dynamic and reversible modification. Deconjugation of SUMO from target proteins is regulated by SUMO proteases (Mukhopadhyay and Dasso, 2007). Ulp/SENP is a family of cysteine proteases that cleave the isopeptide bond between SUMO and its target protein. These enzymes are also responsible for maturation of newly synthesized SUMO prior to conjugation in order to expose a C-terminal GlyGly motif (removing one amino acid, phenylalanine, in C. elegans SMO-1). There are four SUMO proteases in C. elegans: ULP-1, ULP-2, ULP-4 and ULP-5 (ULP-3 is the putative Nedd8 protease ortholog) (Sapir et al., 2014), their specific functions have been started to be elucidated recently (Pelisch et al., 2014; Sapir et al., 2014; Tsur et al., 2015). ULP-4 was shown to have a role during the first mitotic division in embryos, ulp-4(RNAi) led to multiple defects in chromosome alignment, spindle rotation and a delay in mitotic exit, however kinetochore proteins were normally recruited (Pelisch et al., 2014). During larval development and adult aging, ULP-4 is localized to the cytosol and mitochondria and regulates the mevalonate pathway by desumoylation of HMG-CoA synthase (HMGS-1), the first enzyme of this pathway. In accordance, ulp-4 mutant worms exhibited phenotypes associated with impaired metabolism such as reduced locomotion and pharyngeal pumping rate and loss of mitochondrial homeostasis (Sapir et al., 2014).

SUMO-interacting motif (SIM). SIMs regulate noncovalent interactions with SUMO and are typically composed of multiple hydrophobic residues and an acidic residue. SIM-containing proteins can be recruited to sumoylated proteins and this may stimulate their own covalent sumoylation. SIMs can also regulate the recruitment of UBC9 that is loaded with SUMO to the protein, resulting a in its covalent sumoylation (Gareau and Lima, 2010). There is no yet functional evidence for SIMs in C. elegans or to an ortholog of the SUMO-targeted ubiquitin E3 ligase, RNF4 (Sun et al., 2007).

\section{SUMO functions in C. elegans}

\section{Initial observations}

The first genome wide RNAi screen in C. elegans for embryonic lethality (chromosome I) identified the $C$. elegans SUMO as required for viability (Fraser et al., 2000). Initial analysis of the SUMO system was done in Candido lab in 2002 (Jones et al., 2002). RNAi to $u b c-9$ or $s m o-1$ resulted in embryonic arrest while less affected animals showed abnormal everted vulva and tail defects. Analysis of microarray data showed high expression of smo-1 during periods of increased cell divisions i.e. embryogenesis and the fourth larval stage and young adults (germline development)(Jones etal., 2002). One of the early studies that demonstrated the conservation of the SUMO system in $C$. elegans was on SOP-2 sumoylation (Zhang et al., 2004). SOP-2 encodes a SAM domain-containing protein localized to nuclear bodies (Zhang et al., 2003) and required for maintaining a restricted pattern of Hox gene expression. A yeast two-hybrid $(\mathrm{Y} 2 \mathrm{H})$ screen revealed its interaction with UBC-9, mediated through the SAM domain. Sumoylation is required for localization of SOP-2 to distinct nuclear bodies. In ubc-9(RNAi) or smo-1 (RNAi) animals an ectopic expression of the HOX genes egl-5 and mab-5 was detected as was observed in sop-2 mutant animals (Zhang et al., 2003; Zhang et al., 2004) thus the SUMO machinery is required for the controlled repression of HOX genes during development.

Characterization of homozygous smo-1 deletion mutant, smo1(ok359), revealed that due to maternal contribution of the smo-1 gene product these mutant animals develop into sterile adults with abnormal somatic gonad, germ line, and vulva (Broday et al., 2004). A specific substrate for SUMO highlighted in this study was the LIM homeodomain transcription factor LIN-11. Mutant smo-1 animals lack a vulval-uterine connection (UTSE) as a result of impaired ventral uterine $\pi$-cell differentiation and anchor cell fusion. This phenotype highly resembles lin-11 mutant phenotype and suggests that LIN-11 function is regulated by sumoylation. LIN-11 expression levels and nuclear localization are impaired in smo-1 mutant worms. Expression of a SUMO-modified LIN-11 (linear fusion that mimics sumoylation (Ross et al., 2002)) in the smo-1 mutant background partially rescued $\pi$-cell differentiation and retained LIN-11 in nuclear bodies. The SUMO acceptor sites on LIN-11 were mapped to Lysine 17 and 18. Expression of transgenes harboring mutations on these SUMO acceptor sites of LIN-11 showed rescue of the vulval defects of lin-11 mutants, however, the UTSE was not normally formed. Thus sumoylation of LIN-11 has a tissue specific function that is required for its activity in the uterine $\pi$-cell for the proper formation of the UTSE. This tissue specific function of SUMO in the regulation of a single transcription factor, LIN-11, highlights the potential complexity of this system.

\section{Germline development}

Germline development is abnormal and lead to complete sterility in homozygous animals to smo-1 deletion (Broday et al., 2004). However, probably due to the residual maternal product, germ cells are formed and pachytene-stage meiotic germ cell can be detected. Staining of smo-1 mutant gonads with the spermatogenicspecific SP56 antibody revealed that the structure of these cells is abnormal and that sperm localization in adults is not restricted to the proximal gonad as in WT (Broday et al., 2004).

$A$ requirement for sumoylation during meiosis is conserved across species (Egydio de Carvalho and Colaiácovo, 2006; Nottke et al., In Press). In Saccharomyces cerevisiae, Zip3 is required for the initiation of the synaptonemal complex (SC) assembly and was suggested to act as an E3 SUMO ligase that sumoylates chromosome axis proteins, thus promoting SC polymerization (Cheng et al., 2006) and to bind to double-strand break (DSB) sites that are being repaired with a crossover (Serrentino et al., 2013). Analysis of smo-1 mutant meiotic germ cells revealed impaired SC disassembly and bivalent organization that is elevated in double smo-1; zhp-3 mutants (zhp-3 is the Zip3 ortholog) (Jantsch et al., 2004; Bhalla et al., 2008). DNA DSB repair is essential for crossover 
events. RHINO is one of the proteins required for DSB repair and DNA damage-induced apoptosis (Cotta-Ramusino et al., 2011). Sumoylation of ZTF-8/RHINO is required for its function in DSB repair and DNA damage response also in C. elegans (Kim and Colaiácovo, 2014, 2015). Expression of a non-sumoylated ZTF-8 transgene resulted in the same phenotype as ztf- 8 null animals i.e. reduced fertility and impaired DNA damage repair.

\section{Pharynx development}

The $C$. elegans pharynx is formed during embryogenesis from the ABa and MS blastomeres (Mango, 2007). The T-box transcription factor TBX-2 is specifically required for the development of ABa-derived pharyngeal muscles (Roy Chowdhuri et al., 2006). A Y2H screen identified UBC-9 and GEI-17 as specific interactors of TBX-2. While gei-17(RNAi) resulted in mild pharyngeal defects, arrested ubc-9(RNAi) embryos and larvae exhibited major anterior pharyngeal defects similar to $t b x-2$ mutants as analyzed by DIC and the ceh-22::gfp marker of pharyngeal muscle differentiation. Most pharyngeal muscles that could still be detected in ubc-9(RNAi) animals were the posterior MS-derived muscles which suggest that the SUMO system is specifically critical in the development of Aba-derived pharyngeal muscles and that this regulation is through modification of TBX-2 and possibly additional factors. Additional support for the direct regulation of TBX-2 by sumoylation is the abnormal accumulation of TBX-2::GFP in nuclear bodies in ubc-9(RNAi) embryos in contrast to uniform expression in the nucleoplasm in WT embryos (Roy Chowdhuri et al., 2006). TBX-2 is sumoylated on two sites located in the DNA binding domain and the $\mathrm{C}$-terminal of the protein. Ectopic expression of a downstream target of TBX-2 was detected in ubc-9(RNAi) embryos similarly to tbx-2 mutants, suggesting that sumoylation of TBX-2 is required for its transcriptional repression activity in vivo (Huber et al., 2013). Sumoylation of TBX-2 is also required for its negative autoregulation mechanism (Milton and Okkema, 2015).

\section{Vulval development and chromatin regulation}

Initial analysis of smo-1 mutant worms showed abnormal vulval structures, ectopic vulva induction (multivulva, Muv) and a protruding -vulva phenotype (Pvl), demonstrating that SUMO is required for vulval morphogenesis (for review on vulval development (Sternberg, 2005)). Although vulval invagination appears normal (probably due to the maternal SUMO product), later vulval cell migrations and cell fusions were abnormal leading to abnormal final structure of the vulva. Ectopic vulva was detected in high percentage of animals and interestingly these pseudo-vulva structures were identified at the posterior side, originating from the P8.p cell, which normally remains epidermal and does not form a vulval tissue (Broday et al., 2004).

LIN-1. LIN-1 is an ETSDNA-binding domain protein required for the inhibition of the $1^{\circ}$ vulval cell fate (form the inner parts of the developing vulva) and loss of function mutations of lin-1 cause a Muv phenotype (Ferguson et al., 1987; Miley et al., 2004). LIN-1 is sumoylated on two acceptor sites and sumoylation was shown to mediate its transcriptional repression activity (Leight et al., 2005). Sumoylated LIN-1 interacted with MEP-1, a component of the NuRD transcriptional repression complex, suggesting that this interaction mediates LIN-1 transcriptional repression (Leight et al., 2005). A recent study showed that the SUMO acceptor sites of LIN-1 at its amino-terminus were required for the binding of LIN-
1 to two nucleosome-remodeling enzymes chromatin regulatory factors: SET-6 and RAD-26. The binding was also detected using a translation fusion of SUMO-1::LIN-1 (non-sumoylated mutant) and confirmed that sumoylated LIN-1 recruited these chromatin factors. Genetic analysis identified functional interaction with RAD26 in vulval development; SET-6 may act with sumoylated LIN-1 transiently or in other cells (Leight et al., 2015).

NHR-25. NHR-25 is a nuclear hormone receptor (NHR) and the ortholog of vertebrate SF-1 and LRH-1. As other targets described here, its interaction with SUMO was discovered using the Y2H system. NHR-25 and SMO-1 were shown to genetically interact during vulval development. Sumoylation decreases the transcriptional activity of NHR-25 and its levels in vulval cells to establish a gradient of active NHR-25 required for the correct determination of vulval cell fates (Ward et al., 2013). Importantly, the sumoylation sites on the $C$. elegans NHR-25 are conserved in the vertebrate proteins SF-1 and LRH-1.

Chromatin regulation. The $C$. elegans vulva serves as a model to study chromatin regulation. Three groups of genes called synthetic multivulval (synMuv) genes (class A,B, C), encode for chromatin regulators and inhibit vulval development by antagonizing Ras signaling through inhibition of the expression and secretion of LIN-3/EGF ligand in the hyp7 hypodermal syncytium that is in contact with the vulval precursor cells (VPCs)(Cui et al., 2006; Fay and Han, 2000). Animals mutant for a single gene of any class have a normal vulva, but animals doubly mutant in genes of two classes adopt the wrong cell fate: one or more of the VPCs that should have a $3^{\circ}$ fate which is competent to form vulval tissue but remains epidermal under wild-type conditions, are induced to have a vulval fate, leading to the multivulval (Muv) phenotype. Some of the synMuv genes function through transcriptional repression. A genome-wide RNAi screen to identify synMuv genes highlighted SUMO as a regulator of synMuv function (Poulin et al., 2005). RNAi of either smo-1, ubc-9 or uba-2 (SUMO E1 subunit) induced the Muv phenotype in the three synMuv backgrounds (classes A,B,C). This result suggests that one or more proteins from each of the $A, B, C$ classes are regulated by sumoylation. Along this study the authors found additional function of SUMO in the vulva: animals treated with smo-1 (RNAi) in a background of reduced Ras function, surprisingly increased Muv levels compared to smo-1 (RNAi) in WT background suggesting that SUMO is involved in the regulation of additional pathway in the vulva that is dependent on Ras. Indeed, additional genetic analysis showed that SUMO negatively regulates one or more components of LIN-12/Notch pathway (Poulin et al., 2005). A recent study highlighted a new role for SUMO in preventing misexpression of germline-specific $\mathrm{P}$ granules in somatic cells through the Mec complex (synMuv B class chromatin factors)(Wu et al., 2012). In Drosophila, the MEC complex recognizes SUMO modifications on transcription factors, which enables its recruitment to promoters and subsequent transcriptional repression (Stielow et al., 2008).

BET1. BET 1 is a conserved double bromodomain protein shown to recognize acetyl-lysines on histone tails and to maintain cell fate (Shibata et al., 2010). BET-1 was identified in an RNAi screen for Muv phenotype performed in the background of a balanced smo1 mutant strain (Gee et al., 2013). BET-1 interacts with SMO-1 through its second bromodomain and can be sumoylated in vitro (Gee et al., 2013). Adults harboring a double mutation bet-1;smo-1 exhibit impaired muscle integrity and decrease in muscle myosin 
levels (Fisher et al., 2013). These studies emphasize the direct link between the SUMO system and chromatin complexes in the regulation of LET-60 (RAS)-mediated signaling.

\section{The dosage compensation complex - SUMO modification of a protein complex}

The dosage compensation complex (DCC) in C. elegans binds to both the $\mathrm{X}$ chromosomes of hermaphrodites to reduce transcription by half (Meyer, 2010). RNAi-mediated knockdown of smo-1 disrupted DCC association with the X chromosome and microarray analysis showed increase in expression levels of $\mathrm{X}$-linked genes in smo-1 depleted embryos. Binding of several DCC condensin subunits was severely reduced along the $\mathrm{X}$ chromosome in smo1(RNAi) embryos and these subunits were shown to be modified by SUMO in WT embryos. The authors demonstrated that sumoylation of DCC components is essential for proper dosage compensation assembly but it is not required for initial targeting of this complex to the X chromosome (Pferdehirt and Meyer, 2013). This study emphasizes the principle that multiple proteins of the same complex are sumoylated in order to enhance complex assembly and function, as was shown for DNA repair proteins in response to DNA DSB (Psakhye and Jentsch, 2012)

\section{Epidermal morphogenesis and cytoskeleton regulation}

\section{Intermediate filament assembly}

In a proteomics screen for SUMO targets, a group of both cytosolic and nuclear intermediate filament proteins was identified (Kaminsky et al.). Analysis of the cytoplasmic intermediate filament (clF) protein named IFB-1 revealed that IFB-1 is sumoylated both in vitro and in vivo. Epidermal cIF are assembled at embryonic elongation, the process by which the embryo extends from a bean shape to a long thin worm (Zhang and Labouesse, 2010) and it is possible to follow their assembly in live embryos. Such analysis revealed that sumoylation of IFB-1 is required in order to retain a soluble pool of non-polymerized IFB-1, essential for its subsequent assembly into the filament and its proper function in embryonic elongation and in the maintenance of muscle attachment to the cuticle. In the absence of SUMO, IFB-1 formed ectopic filaments and protein aggregates. Soluble IFB-1 could be detected in WT embryos at early elongation while in smo-1(RNAi) embryos abnormal thick and short filaments were accumulated (Kaminsky et al.). Another study showed that knockdown of $u b c-9$ gave the same abnormal IF assembly phenotypes in embryos (Lim et al., 2014). It is possible that in addition to SUMO function as a sequestering factor, sumoylated IFB-1 binds accessory, regulatory and polymerization factors that are required for normal filament assembly. When SUMO is depleted this pool is eliminated which leads to aberrant polymerization of IFB-1 immediately following their translation. Fluorescence recovery after photobleaching (FRAP) showed that depletion of SUMO or mutation of the SUMO acceptor site on IFB-1 resulted in a reduction of IFB1 cytoplasmic soluble pool. This mutation on the sumoylation site impairs IFB-1 activity in embryonic elongation (Kaminsky et al.). A regulated desumoylation process may be responsible for the ability of the free IFB-1 subunits to polymerize into the mature filaments.

\section{The cadherin-catenin complex}

In order to determine if transient sumoylation contributes to the regulation of dynamic morphogenetic events, we are currently dis- secting the functions of the four $C$. elegans SUMO proteases. It is still unknown what are their targets, functional differences and mode of regulation of these enzymes. Knockdown of the SUMO protease ULP-2 caused embryonic arrest during epidermal morphogenesis. The $C$. elegans epidermal adherens junctions (AJs) were found to be the key target for ULP-2 activity at this developmental stage. AJs consist of E-cadherin transmembrane adhesion molecules linked to cytoplasmic structural and regulatory proteins forming stable adhesive contacts between cells. AJs mediate both the rigidity and plasticity of cell-cell contacts required during morphogenesis. HMR-1/E-cadherin is sumoylated and is a substrate for ULP-2 SUMO protease activity. In addition the results showed that (1) sumoylation of HMR-1/E-cadherin cytoplasmic tail impairs its binding to HMP-2/ $\beta$-catenin and therefore its interaction with the actin cytoskeleton, and (2) continuous sumoylation and desumoylation of $\mathrm{HMR}-1 / \mathrm{E}$-cadherin regulates its recruitment and function at the apical AJs. These observation highlight the SUMO system as a molecular switch that modulates the binding of E-cadherin to the actin cytoskeleton (Tsur et al., 2015).

\section{Cilia}

Another evidence for the role of SUMO in cytoskeleton organization is the sumoylation of the small GTPase ARL-13, the worm orthologue of $A R L 13 B$ (mutated in ciliopathy Joubert syndrome) ( $\mathrm{Li}$ et al., 2012). ARL-13 plays a role in ciliogenesis and its sumoylation is required for its function in ciliary targeting of sensory receptors but not for its targeting to cilia. Expression of ARL-13 chimera protein with SMO-1 (mimics constitutively sumoylated ARL-13) could rescue the ciliogenesis defects of arl-13 mutant worms. The authors also found that UBC-9, in addition to its known nuclear localization is localized to the middle segments of cilia, similarly to $A R L-13$ and that the human ortholog ARL13B is also sumoylated, proving functional conservation of sumoylation of these ciliary protein (Li et al., 2012).

\section{Additional substrates for SUMO and possible cross talk with other post translational modifications}

Two components of the SUMO system, UBA-2 and GEI-17 were identified in an RNAi -based genome-wide screen for synthetic lethality performed with conditional mutants of the PAR-1 and PAR-4 polarity proteins (Morton et al., 2012). This suggests that sumoylation may be important for polarity or PAR protein function in $C$. elegans. However, since strong polarity defects were not observed in early embryos, the authors suggested that the role of the SUMO system in polarity could be later in development following the two-cell stage through regulation of cytoskeletal proteins (Morton et al., 2012).

A few more examples of identified SUMO targets in C. elegans are the ER stress responsive transcription factor XBP-1 (Lim et al., 2014), the nuclear AAA ATPase FIGL-1/Fidgetin (Onitake et al., 2012) and BRD-1, the ortholog of BARD1 (BRCA1-associated RING domain 1) (Boulton et al., 2004). As detailed above, many targets of SUMO were identified using the $\mathrm{Y} 2 \mathrm{H}$ system. In a proteomics screen for SUMO targets, we identified $\sim 250$ putative targets in $C$. elegans (Kaminsky et al.). In addition to the expected high fraction of nuclear proteins we identified a large group of cytosolic, membrane, and other subcellular organelle proteins. An additional group included cytoskeleton components: actin-binding proteins, myosins, $\alpha$ - and $\beta$-tubulin and intermediate filament proteins. An 
interesting group of putative targets were proteins involved in posttranslational modifications such as ubiquitination, phosphorylation, glycosylation and myristoylation. This highlights possible cross-talk between sumoylation and various post-translational modification pathways (the cross-talk with ubiquitin is well established; Gareau and Lima, 2010). Advanced mass spectrometry methods have been developed in the last few years that allow identification of SUMO substrates as well as the sumoylation site/s on each target (Hendriks and Vertegaal, 2016).

\section{Summary}

Sumoylation is involved in multiple cellular processes. Here I summarized the main findings about SUMO functions in C. elegans. Proteomics and transcriptomic data suggest that SUMO has additional functions and targets during development yet to be elucidated. Sumoylation affects each substrate in a unique way by changing interactions with cellular macromolecules. Sumoylation introduce a major alteration to the substrate that changes its activity or localization dramatically. It is still unknown how this system is regulated. The advanced tools developed for $C$. elegans research together with the extensive knowledge gained on the biology of this multicellular organism will allow us to better decode how this reversible modification regulates different cell types and tissues during animal development from embryogenesis to adults.

\section{Acknowledgments}

I sincerely apologize to those people whose work could not be cited owing to space limitations. Work in my laboratory is supported by grants from the Israel Science Foundation (ISF 980/06, 1617/11 and 1878/15) and the Israel Cancer Research Fund (06-203-RCDA, PG-11-3086 and $P G-14-101)$.

\section{Reference}

BHALLA, N., WYNNE, D.J., JANTSCH, V., DERNBURG, A.F. (2008) ZHP-3 Acts at Crossovers to Couple Meiotic Recombination with Synaptonemal Complex Disassembly and Bivalent Formation in C. elegans. PLoS Genet 4: e1000235.

BOULTON, S.J., MARTIN, J.S., POLANOWSKA, J., HILL, D.E., GARTNER, A., VIDAL, M. (2004) BRCA1/BARD1 Orthologs Required for DNA Repair in Caenorhabditis elegans. Curr. Biol. 14: 33-39.

BRODAY, L., KOLOTUEV, I., DIDIER, C., BHOUMIK, A., GUPTA, B.P., STERNBERG, P.W., PODBILEWICZ, B., RONAI, Z.E. (2004) The small ubiquitin-like modifier (SUMO) is required for gonadal and uterine-vulval morphogenesis in Caenorhabditis elegans. Genes Dev. 18: 2380-2391.

CHENG, C.-H., LO, Y.-H., LIANG, S.-S., TI, S.-C., LIN, F.-M., YEH, C.-H., HUANG, H.-Y., WANG, T.-F. (2006) SUMO modifications control assembly of synaptonemal complex and polycomplex in meiosis of Saccharomyces cerevisiae. Genes Dev. 20: 2067-2081.

COTTA-RAMUSINO, C., MCDONALD, E.R., HUROV, K., SOWA, M.E., HARPER, J.W., ELLEDGE, S.J. (2011) A DNA Damage Response Screen Identifies RHINO, a 9-1-1 and TopBP1 Interacting Protein Required for ATR Signaling. Science 332: 1313-1317.

CUI, M., CHEN, J., MYERS, T.R., HWANG, B.J., STERNBERG, P.W., GREENWALD, I., HAN, M. (2006) SynMuv Genes Redundantly Inhibit lin-3/EGF Expression to Prevent Inappropriate Vulval Induction in C. elegans. Dev. Cell 10: 667-672.

EGYDIO DE CARVALHO, C., COLAIÁCOVO, M.P. (2006) SUMO-mediated regulation of synaptonemal complex formation during meiosis. Genes Dev. 20: 1986-1992.

FAY, D.S., HAN, M. (2000) The synthetic multivulval genes of $C$. elegans: functional redundancy, Ras-antagonism, and cell fate determination. Genesis 26: 279-284.

FERGUSON, E.L., STERNBERG, P.W., HORVITZ, H.R. (1987) A genetic pathway for the specification of the vulval cell lineages of Caenorhabditis elegans. Nature 326: 259-267.
FERREIRA, H.C., TOWBIN, B.D., JEGOU, T., GASSER, S.M. (2013) The shelterin protein POT-1 anchors Caenorhabditis elegans telomeres through SUN-1 at the nuclear periphery. J. Cell Biol. 203: 727-735.

FISHER, K., GEE, F., WANG, S., XUE, F., KNAPP, S., PHILPOTT, M., WELLS, C., RODRIGUEZ, M., SNOEK, L.B., KAMMENGA, J., POULIN, G.B. (2013) Maintenance of muscle myosin levels in adult $C$. elegans requires both the double bromodomain protein BET-1 and sumoylation. Biol. Open 2: 1354-1363.

FLOTHO, A., MELCHIOR, F. (2013) Sumoylation: A Regulatory Protein Modification in Health and Disease. Annu.Rev. Biochem. 82: 357-385.

FRASER, A.G., KAMATH, R.S., ZIPPERLEN, P., MARTINEZ-CAMPOS, M., SOHRMANN, M., AHRINGER, J. (2000) Functional genomic analysis of $C$. elegans chromosome I by systematic RNA interference. Nature 408: 325-330.

GAREAU, J.R., LIMA, C.D. (2010) The SUMO pathway: emerging mechanisms that shape specificity, conjugation and recognition. Nat Rev Mol Cell Biol 11: 861-871.

GEE, F., FISHER, K., KLEMSTEIN, U., POULIN, G.B. (2013) An RNAi-Based Dimorphic Genetic Screen Identified the Double Bromodomain Protein BET-1 as a Sumo-Dependent Attenuator of RAS-Mediated Signalling. PLoS ONE 8: e83659.

HENDRIKS, I.A., VERTEGAAL, A.C.O. (2016) Acomprehensive compilation of SUMO proteomics. Nat Rev Mol Cell Biol 17: 581-595.

HOLWAY, A.H., HUNG, C., MICHAEL, W.M. (2005) Systematic, RNA-InterferenceMediated Identification of mus-101Modifier Genes in Caenorhabditis elegans. Genetics 169: 1451-1460.

HUBER, P., CRUM, T., CLARY, L.M., RONAN, T., PACKARD, A.V., OKKEMA, P.G. (2013) Function of the $C$. elegans T-box factor TBX-2 depends on SUMOylation. Cell. Molec. Life Sci. 70: 4157-4168.

JANTSCH, V., PASIERBEK, P., MUELLER, M.M., SCHWEIZER, D., JANTSCH, M., LOIDL, J. (2004) Targeted Gene Knockout Reveals a Role in Meiotic Recombination for ZHP-3, a Zip3-Related Protein in Caenorhabditis elegans. Molec. Cell. Biol. 24: 7998-8006.

JONES, D., CROWE, E., STEVENS, T.A., CANDIDO, E.P. (2002) Functional and phylogenetic analysis of the ubiquitylation system in Caenorhabditis elegans: ubiquitin-conjugating enzymes, ubiquitin-activating enzymes, and ubiquitin-like proteins. Genome Biol 3: RESEARCH0002.

Kaminsky, R., Denison, C., Bening-Abu-Shach, U., Chisholm, A.D., Gygi, S.P., Broday, L. SUMO Regulates the Assembly and Function of a Cytoplasmic Intermediate Filament Protein in C. elegans. Dev. Cell 17: 724-735.

KIM, H.-M., COLAIÁCOVO, M.P. (2014) ZTF-8 Interacts with the 9-1-1 Complex and Is Required for DNA Damage Response and Double-Strand Break Repair in the C. elegans Germline. PLoS Genet 10: e1004723.

KIM, H.-M., COLAIÁCOVO, M.P. (2015) New Insights into the Post-Translational Regulation of DNA Damage Response and Double-Strand Break Repair in Caenorhabditis elegans. Genetics 200: 495-504.

KIM, S.-H., MICHAEL, W.M. (2008) Regulated Proteolysis of DNA Polymerase $\eta$ during the DNA-Damage Response in C. elegans. Molec. Cell 32: 757-766.

LEIGHT, E.R., GLOSSIP, D., KORNFELD, K. (2005) Sumoylation of LIN-1 promotes transcriptional repression and inhibition of vulval cell fates. Development 132: 1047-1056.

LEIGHT, E.R., MURPHY, J.T., FANTZ, D.A., PEPIN, D., SCHNEIDER, D.L., RATLIFF, T.M., MOHAMMAD, D.H., HERMAN, M.A., KORNFELD, K. (2015) Conversion of the LIN-1 ETS Protein of Caenorhabditis elegans from a SUMOylated Transcriptional Repressor to a Phosphorylated Transcriptional Activator. Genetics 199: 761-775.

LI, Y., ZHANG, Q., WEI, Q., ZHANG, Y., LING, K., HU, J. (2012) SUMOylation of the small GTPase ARL-13 promotes ciliary targeting of sensory receptors. J. Cell Biol. 199: 589-598.

LIM, Y., LEE, D., KALICHAMY, K., HONG, S.-E., MICHALAK, M., AHNN, J., KIM, D.H., LEE, S.-K. (2014) Sumoylation regulates ER stress response by modulating calreticulin gene expression in XBP-1-dependent mode in Caenorhabditis elegans. Int. J. Biochem. Cell Biol. 53: 399-408.

MANGO, S.E. (2007) The C. elegans pharynx: a model for organogenesis. WormBook, 1-26.

MEYER, B.J. (2010) Targeting X chromosomes for repression. Curr. Opin. Genet. Dev. 20: 179-189.

MILEY, G.R., FANTZ, D., GLOSSIP, D., LU, X., SAITO, R.M., PALMER, R.E., INOUE, T., VANDEN HEUVEL, S., STERNBERG, P.W., KORNFELD, K. (2004) Identification of Residues of the Caenorhabditis elegans LIN-1 ETS Domain That Are Necessary for DNA Binding and Regulation of Vulval Cell Fates. Genetics 167: 1697-1709. 
MILTON, A.C., OKKEMA, P.G. (2015) Caenorhabditis elegans TBX-2 Directly Regulates Its Own Expression in a Negative Autoregulatory Loop. G3: Genes Genomes Genet. 5: 1177-1186.

MORTON, D.G., HOOSE, W.A., KEMPHUES, K.J. (2012) A Genome-Wide RNAi Screen for Enhancers of Mutants Reveals New Contributors to Early Embryonic Polarity in Caenorhabditis elegans. Genetics 192: 929-942.

MUKHOPADHYAY, D., DASSO, M. (2007) Modification in reverse: the SUMO proteases. Trends Biochem. Sci. 32: 286-295.

NOTTKE, A., HYUN-MIN K., AND COLAIÁCOVO, M.P. (2016). Wrestling with chromosomes: The roles of SUMO during meiosis. In SUMO regulation of cellular processes. (ed. V.G. Wilson), In Press. Springer Publishing.

ONITAKE, A., YAMANAKA, K., ESAKI, M., OGURA, T. (2012) Caenorhabditis elegans fidgetin homolog FIGL-1, a nuclear-localized AAA ATPase, binds to SUMO. J. Struct. Biol. 179: 143-151.

PELISCH, F., SONNEVILLE, R., POURKARIMI, E., AGOSTINHO, A., BLOW, J.J., GARTNER, A., HAY, R.T. (2014) Dynamic SUMO modification regulates mitotic chromosome assembly and cell cycle progression in Caenorhabditis elegans. Nat Commun 5: 5485

PFERDEHIRT, R.R., MEYER, B.J. (2013) SUMOylation is essential for sex-specific assembly and function of the Caenorhabditis elegans dosage compensation complex on X chromosomes. Proc. Natl. Acad. Sci. USA 110: E3810-E3819.

POULIN, G., DONG, Y., FRASER, A.G., HOPPER, N.A., AHRINGER, J. (2005) Chromatin regulation and sumoylation in the inhibition of Ras-induced vulval development in Caenorhabditis elegans. EMBO J. 24: 2613-2623.

PSAKHYE, I., JENTSCH, S. (2012) Protein Group Modification and Synergy in the SUMO Pathway as Exemplified in DNA Repair. Cell 151: 807-820.

ROERINK, S.F., KOOLE, W., STAPEL, L.C., ROMEIJN, R.J., TIJSTERMAN, M. (2012) A Broad Requirement for TLS Polymerases $\eta$ and $\mathrm{K}$, and Interacting Sumoylation and Nuclear Pore Proteins, in Lesion Bypass during C. elegans Embryogenesis. PLoS Genet 8: e1002800.

ROSS, S., BEST, J.L., ZON, L.I., GILL, G. (2002) SUMO-1 Modification Represses Sp3 Transcriptional Activation and Modulates Its Subnuclear Localization. Molec. Cell 10: 831-842.

ROY CHOWDHURI, S., CRUM, T., WOOLLARD, A., ASLAM, S., OKKEMA, P.G. (2006) The T-box factor TBX-2 and the SUMO conjugating enzyme UBC-9 are required for ABa-derived pharyngeal muscle in C. elegans. Dev. Biol. 295:664-677.

RYTINKI, M.M., LAKSO, M., PEHKONEN, P., AARNIO, V., REISNER, K., PERÄKYLÄ, M., WONG, G., PALVIMO, J.J. (2011) Overexpression of SUMO perturbs the growth and development of Caenorhabditis elegans. Cell. Molec. Life Sci. 68:3219-3232.
SAPIR, A., TSUR, A., KOORMAN, T., CHING, K., MISHRA, P., BARDENHEIER, A., PODOLSKY, L., BENING-ABU-SHACH, U., BOXEM, M., CHOU, T.-F., BRODAY, L., STERNBERG, P.W. (2014) Controlled sumoylation of the mevalonate pathway enzyme HMGS-1 regulates metabolism during aging. Proc. Natl. Acad. Sci. USA 111: E3880-E3889.

SERRENTINO, M.-E., CHAPLAIS, E., SOMMERMEYER, V., BORDE, V. (2013) Differential Association of the Conserved SUMO Ligase Zip3 with Meiotic Double-Strand Break Sites Reveals Regional Variations in the Outcome of Meiotic Recombination. PLoS Genetics 9: e1003416.

SHIBATA, Y., TAKESHITA, H., SASAKAWA, N., SAWA, H. (2010) Double bromodomain protein BET-1 and MYST HATs establish and maintain stable cell fates in C. elegans. Development 137: 1045-1053.

STERNBERG, P.W. (2005) Vulval development. WormBook, ed. The C. elegans Research Community, WormBook, doi/10.1895/wormbook.1.6.1, http://www. wormbook.org

STIELOW, B., SAPETSCHNIG, A., KRÜGER, I., KUNERT, N., BREHM, A., BOUTROS M., SUSKE, G.(2008) Identification of SUMO-Dependent Chromatin-Associated Transcriptional Repression Components by a Genome-wide RNAi Screen. Molec. Cell 29: 742-754.

SUN, H., LEVERSON, J.D., HUNTER, T. (2007) Conserved function of RNF4 family proteins in eukaryotes: targeting a ubiquitin ligase to SUMOylated proteins. EMBO J. 26: 4102-4112

TSUR, A., BENING ABU-SHACH, U., BRODAY, L. (2015) ULP-2 SUMO Protease Regulates E-Cadherin Recruitment to Adherens Junctions. Dev. Cell 35: 63-77.

WARD, J.D., BOJANALA, N., BERNAL, T., ASHRAFI, K., ASAHINA, M., YAMAMOTO K.R. (2013) Sumoylated NHR-25/NR5A Regulates Cell Fate during C. elegans Vulval Development. PLoS Genet 9: e1003992.

WU, X., SHI, Z., CUI, M., HAN, M., RUVKUN, G. (2012) Repression of Germline RNA Pathways in Somatic Cells by Retinoblastoma Pathway Chromatin Complexes. PLoS Genet 8: e1002542.

ZHANG, H., AZEVEDO, R.B.R., LINTS, R., DOYLE, C., TENG, Y., HABER, D., EMMONS, S.W. (2003) Global Regulation of Hox Gene Expression in C. elegans by a SAM Domain Protein. Dev. Cell 4: 903-915

ZHANG, H., LABOUESSE, M. (2010) The making of hemidesmosome structures in vivo. Dev. Dynam. 239: 1465-1476.

ZHANG, H., SMOLEN, G.A., PALMER, R., CHRISTOFOROU, A., VAN DEN HEUVEL, S., HABER, D.A. (2004) SUMO modification is required for in vivo Hox gene regulation by the Caenorhabditis elegans Polycomb group protein SOP-2. Nat Genet 36: 507-511. 


\section{Further Related Reading, published previously in the Int. J. Dev. Biol.}

Histone deacetylases 1 and 2 are required for brain development Joanna Jaworska, Malgorzata Ziemka-Nalecz and Teresa Zalewska Int. J. Dev. Biol. (2015) 59: 171-177

https://doi.org/10.1387/ijdb.150071tz

a integrin cytoplasmic tails have tissue-specific roles during C. elegans development Christopher M. Meighan and Jean E. Schwarzbauer

Int. J. Dev. Biol. (2014) 58: 325-333

https://doi.org/10.1387/ijdb.130327cm

Differences in embryonic pattern formation between Caenorhabditis elegans and its close parthenogenetic relative Diploscapter coronatus

Vera Lahl, Jens Schulze and Einhard Schierenberg

Int. J. Dev. Biol. (2009) 53: 507-515

https://doi.org/10.1387/ijdb.082718vl

The embryonic cell lineage of the nematode Rhabditophanes sp.

Wouter Houthoofd, Maxime Willems, Kim Jacobsen, August Coomans and Gaetan Borgonie Int. J. Dev. Biol. (2008) 52: 963-967

https://doi.org/10.1387/ijdb.072404wh

Genes required for the common miracle of fertilization in Caenorhabditis elegans Andrew Singson, Julie S. Hang and Jean M. Parry Int. J. Dev. Biol. (2008) 52: 647-656

https://doi.org/10.1387/ijdb.072512as

Egg development in parthenogenetic nematodes: variations in meiosis and axis formation Vera Lahl, Bernd Sadler and Einhard Schierenberg Int. J. Dev. Biol. (2006) 50: 393-397

https://doi.org/10.1387/ijdb.052030vl

\section{Worm tales}

$J$ White

Int. J. Dev. Biol. (2000) 44: 39-42

http://www.intjdevbiol.com/web/paper/10761845
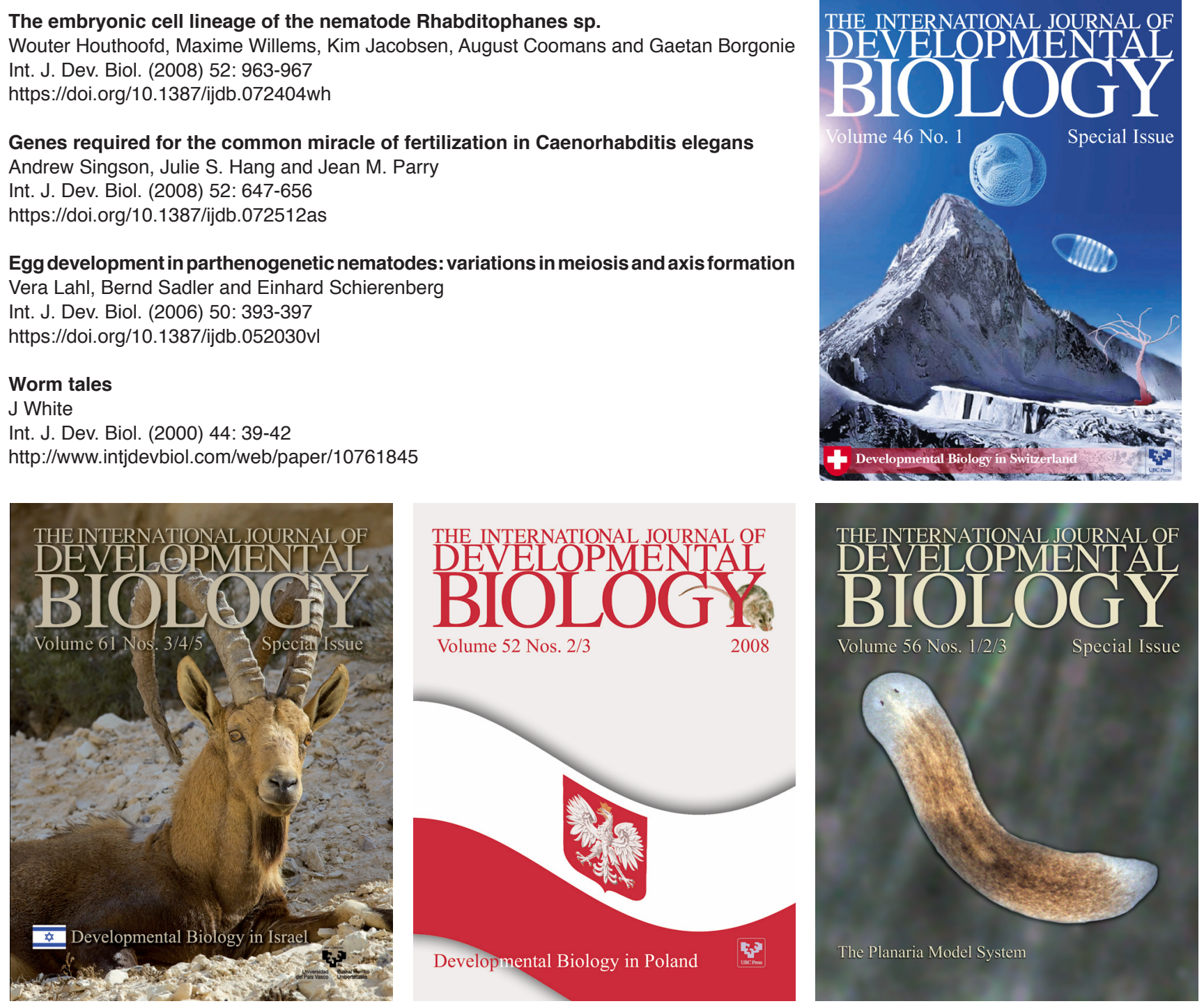